\title{
An Interesting Case Report of Frontal Sinus Keratocyst
}

\author{
Alireza Mohebbi ${ }^{1} \&$ Mohammad Aghajanpour ${ }^{2}$ \\ ${ }^{1}$ ENT and Head and Neck Research Center, Hazrat Rasoul Hospital, The Five Senses Institute, Iran University \\ of Medical Sciences, Tehran, Iran \\ ${ }^{2}$ Ghods Hospital, Arak, Iran \\ Correspondence: Mohammad Aghajanpour, Ghods Hospital, Arak, Iran. E-mail: Aghajanpour560@yahoo.com
}

Received: May 20, 2019

Accepted: September 26, 2019

Online Published: October 23, 2019

doi:10.5539/jmbr.v9n1p100

URL: https://doi.org/10.5539/jmbr.v9n1p100

\begin{abstract}
Purpose: To report an unusual case of frontal sinus keratocyst.

Case Report: A 31 year old woman presented with a history of swollen right eye and visual field impairment. The ophthalmological examination revealed right superior eyelid edema and some degrees of proptosis. CT scan with iodine injection evidenced the opacification of the right frontal sinus with superior orbital wall lysis. Complete endoscopic surgical resection of a mass containing foul smelling keratin material was performed via Draf type III. The patient became symptomatic after 6 years. Imaging studies showed neo- osteogenesis in the far lateral end of right frontal sinus. Frontal trephination was done and the remnant of keratocyst was removed and the septum interfacing the cell and the right frontal sinus was walled down entirely.

Conclusion: Although frontal sinus keratocyst is benign but can spread to the surrounding structures leading to several complications. Therefore, complete surgical resection is necessary to avoid recurrence.
\end{abstract}

Keywords: Keratocyst, Frontal Sinus

\section{Introduction}

Keratocyst or more accurately known keratoma or cholesteatoma (Hopp \& Montgomery, 1984; Passke, 1984) is a mass composed of keratin layers within hyper-keratinized squamous epithelium that replaces normal mucus membrane (Rand \& Reeves, 1943). It is generally a pathology of middle ear cavity and mastoids, but has been described in skin, mandible, orbit and central nervous system. When it is drained, thick cheesy keratin debris along with purulent material with foul smelling is expressed (Hopp \& Montgomery, 1984).

Although cholesteatoma of the paranasal sinuses is uncommon but makes the differential diagnosis of unilateral sinus masses interesting. In English literature, about 30 cases of paranasal sinus cavity cholesteatoma have been reported (Hartman \& Stankiewicz, 1991).

The most common paranasal sinus cholesteatoma is frontal sinus, followed by maxillary and ethmoid sinuses (Calcaterra \& Schwartz, 1976).

The first paranasal sinus cholesteatoma has been reported by Hutcheon in 1941 which was a case of rhinolith combined with cholesteatoma of the maxillary antrum (Hutcheon, 1941).

Spencer was the first one who reported a frontal sinus cholesteatoma in 1930 (Spencer, 1930).

It has been pointed that bone tissue is the key link in the phosphoric-calcium homeostasis system, which can be maintained through the multilevel physiological systems (Domenyuk, Zelensky, Rzhepakovsky, \& Anfinogenova, 2018). A surgical procedure can be considered as the last treatment option for patients who have degenerative changes (AlAzzam, Alaskar, AlRabiah, Alhaqbani, AlFaleh, AlMugren, AlAzzam, \& AlQahtani, 2018). Lesion form is also an effective factor on observing it in radiography stereotype (Siahtiri, Mehrabi, Ghorani, \& Mohagheghi, 2018).

\section{Case Presentation}

A 31 year old Iranian woman, otherwise fit and healthy, presented with a history of right-sided painful supraorbital tumefaction experiencing a minor visual field impairment and some degrees of double vision. There was a history of frontal cephalgia and right-sided facial pain and eyelid edema. There was neither history of external eye and frontal trauma, nor previous sinonasal or ophthalmological operation. A low grade fever (38/1C) 
was detected. The Glasgow score was 15/15.

The ophthalmological examination revealed significant superior palpebral edema and a slight right-sided proptosis with restriction of upward gaze. Visual acuity was normal. The colour vision was intact and there was no afferent pupillary defect. Nasal endoscopy was unremarkable, apart from some congestion at the right middle meatus. The computerized tomography (CT) scan showed a soft tissue lesion of low density that totally filled the right frontal sinus, eroding through the orbital roof and displacing the right globe (Figure 1).

The anterior and posterior frontal tables were intact. The patient underwent right-sided endoscopic sinus surgery. A transeptal frontal sinusotomy or median frontal drainage procedure (Draf III) was done for the patient.

Intra-operatively, the right frontal sinus was found to be full of keratin material. The frontal sinus was thoroughly debrided as far as possible. There was a bone lysis of the inferior wall of right frontal sinus exposing the superior roof of the right orbit. The histopathologic examination showed a fibrocollagenous wall lined with squamous epithelium, containing lamellar layers of keratin known as a keratocyst or cholesteatoma. The patient remained disease free at 5 years follow-up, but in the $6^{\text {th }}$ year of follow-up, the patient experienced several episodes of right fronto-temporal headache, superior eyelid edema, painful right globe movements with conjunctivitis and cilliary injection.

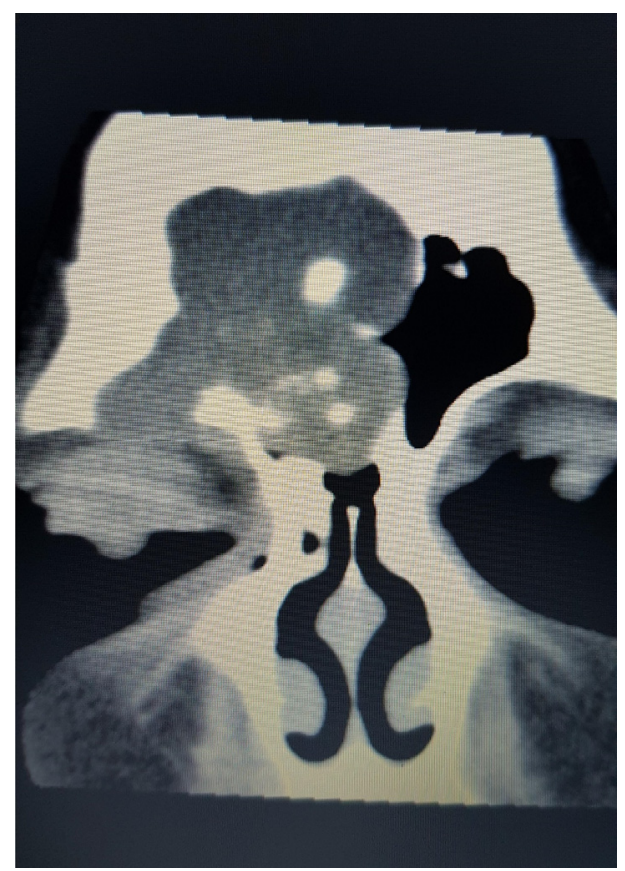

Figure 1. Coronal view prior to the 1st surgery

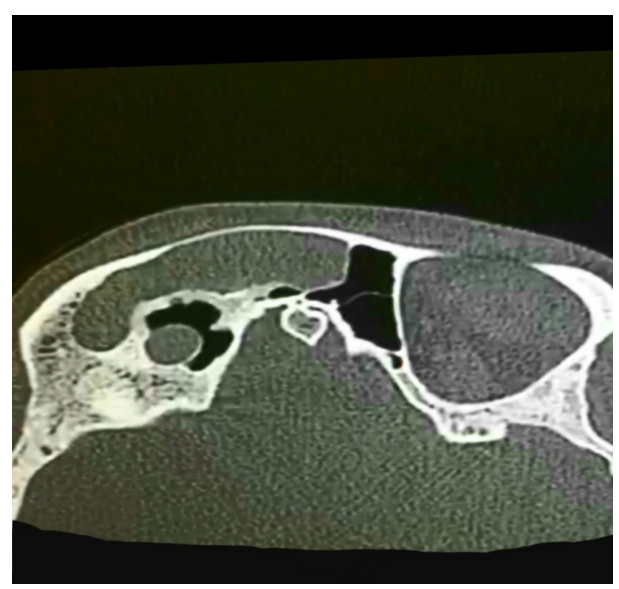

Figure 2. Axial view before the revision surgery. Neo-osteogenesisis formed due to keratocyst remnant 


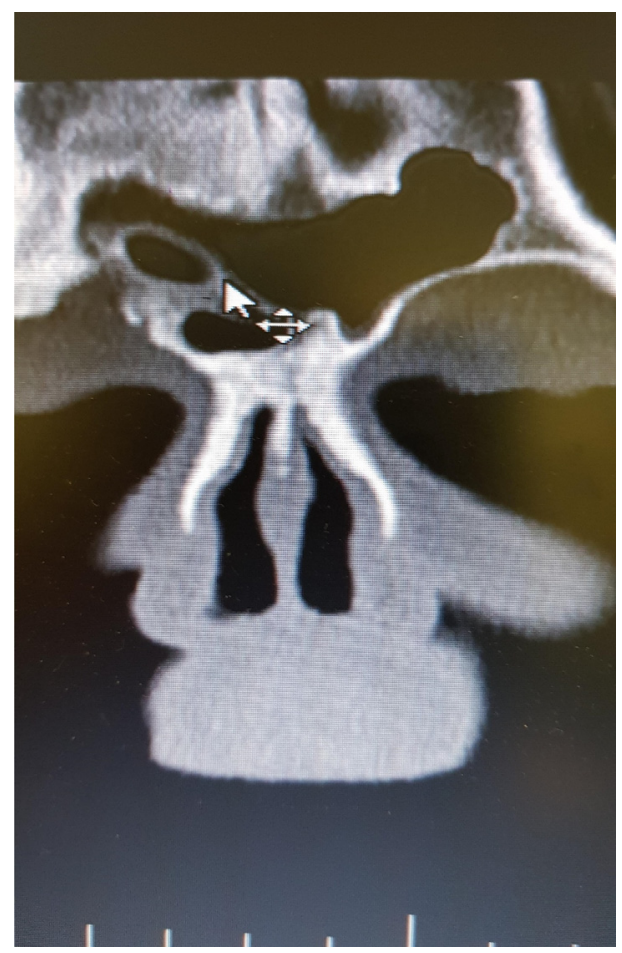

Figure 3. Neo-osteogenesis in the far lateral end of Rt. Frontal sinus due to the keratocyst

Finally, a CT scan has been done, which showed an opacification just in the supero-lateral part of the right orbit, definitely in the terminal lateral end of the right frontal sinus which was separated from the frontal sinus by a thick septum as a result of neo-osteogenesis, resemblibg a new chamber in the far lateral end of the right frontal sinus; filled with non-enhancing lesion and with orbital extension causing gross downward and outward displacement of the globe (Figures 2, 3, 4). At the same time, erosion of the roof and supero-lateral wall of the right orbit has been noticed. Posteriorly, MRI of paranasal sinuses and orbit was suggested to better define the lesion. MRI showed an isolated iso-intense superior orbital cell arising from the far lateral end of the right frontal sinus causing some degrees of right globe proptosis. Operation has been scheduled for the patient. Image guided technique (navigation) has been contributed to the operation. To reach the cell, an external surgical approach was used, through an incision in the superolateral part of the right eyebrow, defined by navigation previously, trephination has been done by a microdrill and a cutting water-refrigerated bur. This approach was used in order to gain better access to the area of the lesion and also to make reconstruction easier. Currettage has been done for extirpation of the lesion.the septum interfacing the frontal sinus and the supra-orbital cell was walled down and the frontal sinus has been extended laterally into the supra-orbital cell. All the secretions were suctioned and complete surgical resection of keratin materials has been done with careful cleansing of sinus cavities with physiological saline. Final histopathological examination established the diagnosis of keratocyst (cholesteatoma) of the right frontal sinus. The incision has been repaired through suture. A post-operative CT scan confirmed the normal expansion of the right frontal sinus.

Post-operatively at 3 months, there was complete resolusion of the patient's signs and symptoms. 


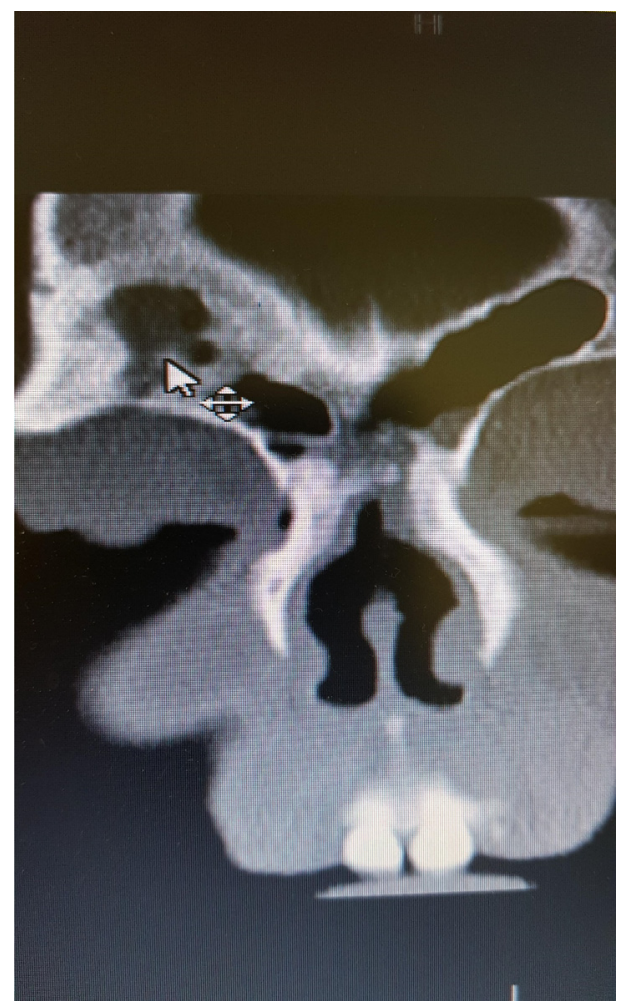

Figure 4. Keratocyst remnant in the Rt. Frontal sinus

\section{Discussion}

Keratocyst or cholesteatoma of paranasal sinuses occurs when the respiratory epithelium that lines the sinus is replaced by hyperkeratotic squamous epithelium which leads to lamellar keratin formation.it is a rare occurance in paranasal sinuses, but most frequently the frontal sinus and more rarely the ethmoidal cells and the maxillary sinus are involved (Campanella, Caldarelli, \& Friedberg, 1979). Mean age of frontal sinus cholesteatoma is 48 , aging from 23 to 80 years (Hansen et al., 2007). Therefore, our case was among the the youngest ones in the literature. keratocysts are classified into primary and secondary types. Primary keratocysts are originated from congenital epithelial cell nests. The secondary cholesteatoma is caused by the migration of keratinizing squamous epithelium in an area where it would not usually be found or implantation beyond its natural boundaries. This could be due to trauma or sequel of any process resulting in sequesteration of normal squamous epithelial cells in abnormal areas (Viswanatha, Nayak, \& Karthik, 2007). But our patient did not experience trauma or other processes that could support the theories. Haeggstrom reported the first case of frontal sinus cholesteatoma in a patient with proptosis in diplopia in 1961 (Haeggstrom, 1961).

It has been pointed that bone tissue is the key link in the phosphoric-calcium homeostasis system, which can be maintained through the multilevel physiological systems. To the best of our knowledge, our patient was the first reported frontal sinus keratocyst (cholesteatoma) in the middle east, and also the first case in Asia. The cholesteatomas are not neoplastic biologically but can erode bones and expand into adjacent areas by enzymes (Amar et al., 1996). Characteristically, sinonasal keratocysts present as a slow-growing unilateral mass usually accompanied by frontal cephalgia, proptosis and mostly without a history of frontal sinus infection by enlargement of keratocysts, bone erosion occurs in all directions (Hammami et al., 2010). Our patient experienced superior eyelid edema, some degrees of proptosis, frontal headache and impaired visual field secondary to the pressure effect on the right globe. At the same time, erosion of right orbital roof has been detected in our patient. CT scan of paranasal sinuses, plays a significant role in the assessment of the disease extension, bone lysis and helps surgical planning. Magnetic resonance imaging (MRI) can be used to differentiate sinusitis from a tumor or a soft tissue lesion (Hutcheon, 1941). Standard management is thorough surgical resection of cholesteatoma (keratocyst) with careful cleansing of sinus cavity (Amar et al., 1996). Complete surgical resection prevents the risk of keratocyst recurrence (Maniglia \& Villa, 1977). In our case, patient became symptomatic again, because of isolation of keratocyst remnant in the far lateral end of right 
frontal sinus. Thus, the curative treatment of frontal sinus keratocyst (cholesteatoma) requires a thorough assessment of paranasal sinuses by CT scanning and MR imaging and then a total excision of keratocyst with adequate drainage, and this approach offers excellent prognosis with the least recurrency rate (Holt, Holt, \& Davis, 1977). In cases of isolated orbital roof defect, as seen in our patient, reconstruction of the orbital roof is not recommended. Lai et al. described successful surgical resection of an extensive frontal sinus keratocyst solely via a modified endoscopic Lothrop (Draf III) (Lai, Liu, \& Chen, 2010).

In our patient, the primary surgery has been done via endoscopic approach (Draf III), but after recurrency, an external approach (frontal trephination) has been used for the revision surgery.

Recently, endoscopic approach with image-guidance had been described (Chandra \& Palmer, 2006). In our case, image-guided technique has been used in the revision surgery. This method provided good access to the area of concern with less morbidity.

Untreated sinus cholesteatoma can lead to carcinomatous degeneration (Osborn \& Wallace, 1967).

\section{Conclusion}

Frontal sinus keratocyst (cholesteatoma) is a rare entity, but should be evoked when a slowly expanding lesion with bone lysis is observed. Pre-operative diagnosis is difficult to establish, because of lack of specificity of clinical presentation and imaging data.

Appropriate treatment of frontal sinus cholesteatoma is complete removal and adequate drainage and sinusotomy for post-operative follow-up to avoid long-term complications.

\section{Conflict of interests}

The authors declare that there is no conflict of interests regarding the publication of this paper.

\section{References}

AlAzzam, S., Alaskar, M. A., AlRabiah, B. A., Alhaqbani, N. M., AlFaleh, A. F., AlMugren, T. S., AlAzzam, A. S., \& AlQahtani, H. M. (2018). Thirty Days' Post-Operative Complications Following Total Knee Replacement in King Abdul-Aziz Medical City, Riyadh Research (Allied Science). International Journal of Pharmaceutical Research \& Allied Sciences, 7(2), 67-73.

Amar et al. (1996). Clinical and biochemical studies of bone destruction in cholesteatoma. J Laryngol Otol., 110, 534-539.

Calcaterra, T. C., \& Schwartz, H. E. (1976). Cholesteatoma of the frontal sinus. Trans Sect Otolaryngol Am Ophthalmol Otolaryngol, 82, 579-81.

Campanella, R. S., Caldarelli, D. D., \& Friedberg, S. A. (1979). Cholesteatoma of the frontal and ethmoid areas. Ann Otol Rhinol Laryngol, 88, 518-23.

Chandra, R. K., \& Palmer, J. N. (2006). Epidermoids of the paranasal sinuses and beyond: Endoscopic management. Am J Rhinol., 20(4), 441-4.

Domenyuk, D. A., Zelensky, V. A., Rzhepakovsky, I. V., \& Anfinogenova, O. I. (2018). Application of Laboratory and X-Ray Gentral Studies un Early Diagnostics of Metabolic Disturbances of Bone Tissue in Children with Autoimmune Diabetes Mellitus. Entomol Appl Sci Lett., 5(4), 1-12.

Haeggstrom, A. A. (1961). Cholesteatoma removed from the frontal sinus. Hygiea, 78, 1122.

Hammami et al. (2010). Cholesteatoma of the frontal sinus. Eur Ann Otorhinolaryngol Head Neck Dis., 127(6), 213-6.

Hansen et al. (2007). Massive cholesteatoma of the frontal sinus: Case report and review of the literature. Auris Nasus Larynx, 34, 387-92.

Hartman, J. M., \& Stankiewicz, J. A. (1991). Cholesteatoma of the paranasal sinuses: Case report and review of the literature. Ear Nose Throat J., 70(10), 719-725.

Holt, G. R., Holt, J. E., \& Davis, W. E. (1977). Late recurrence of a frontal sinus cholesteatoma. Ann. Otol., $86(6 \mathrm{pt} 1), 825$.

Hopp, M. L., \& Montgomery, W. W. (1984). Primary and secondary keratoma of the frontal sinus. Laryngoscope, 94, 628-632.

Hutcheon, J. R. (1941). A case of rhinolith combined with cholesteatoma of the maxillary antrum. Med J Aust., 1 , 451. 
Lai, J. C., Liu, C. K., \& Chen, M. K. (2010). Removal of frontal sinus keratoma solely via endoscopic sinus surgery. J. Layngol. Otol., 124, 1116-9.

Maniglia, A. J., \& Villa, L. (1977). Epidermoid carcinoma of the frontal sinus secondary to cholesteatoma. Trans Sect Otolaryngol Am Acad Ophthalmol Otolaryngol, 84, 112-5.

Osborn, D. A., \& Wallace, M. (1967). Carcinoma of the frontal sinus associated with epidermoid cholesteatoma. J Laryngol Otol., 81(9), 1021-32.

Passke, P. B. (1984). Cholesteatoma of the maxillary sinus( a case report). J Laryngol Otol., 98, 539-541.

Rand, G. W., \& Reeves, D. L. (1943). Dermoid and epidermoid tumours (cholesteatomas) of the central nervous system. Arch. Surg., 46, 350-376.

Siahtiri, S., Mehrabi, A., Ghorani, N., \& Mohagheghi, S. A. (2018). Comparison Of The Diagnostic Accuracy Of Different Image Processing Techniques For Detection Of Bony Lesions In Indirect Digital Radiography. Annals of Dental Specialty, 6(4), 428-432.

Spencer, F. R. (1930). Primary cholesteatoma of the sinuses and orbit: Report of a case of many years and durations followed by carcinoma and death. Arch Otolaryngol, 12, 44-48.

Viswanatha, B., Nayak, L. K., \& Karthik, S. (2007). Cholesteatoma of the maxillary sinus. Ear Nose Throat J., $86,351-353$.

\section{Copyrights}

Copyright for this article is retained by the author(s), with first publication rights granted to the journal.

This is an open-access article distributed under the terms and conditions of the Creative Commons Attribution license (http://creativecommons.org/licenses/by/4.0/). 